\title{
La Red Andaluza de Semillas, buenas prácticas en el mantenimiento sostenible del ecosistema agrario
}

Juan José Soriano Niebla | Red Andaluza de Semillas

URL de la contribución <www.iaph.es/revistaph/index.php/revistaph/article/view/4944>

\section{RESUMEN}

En sus más de 18 años de recorrido, la Red de Andaluza de Semillas ha venido desarrollando y documentando una importante experiencia en la defensa, recuperación y utilización de la biodiversidad cultivada. Esta experiencia ha servido de base para reflexionar sobre la complejidad de este legado rural y las diferentes formas de nombrarlo y entenderlo: recursos genéticos, variedades tradicionales y locales, conocimiento campesino, memoria biocultural... En este extenso patrimonio, de naturaleza tanto cultural como natural, se encuentran claves para conciliar los objetivos de desarrollo con el uso sostenible de los recursos a escala local. Las visiones desarrolladas en los ámbitos de la agroecología y la soberanía alimentaria permiten interpretar esta sabiduría y contextualizarla para dar respuesta a retos actuales. Así han surgido estas buenas prácticas para el cultivo y la elaboración de alimentos, respetuosas con la protección del medio natural y basadas en la valorización del patrimonio genético agrícola.

\section{Palabras clave}

Agroecología | Biodiversidad | Campesinado | Conocimiento | Conservación (patrimonio) | Investigación-acción participativa | Patrimonio agrícola | Red Andaluza de Semillas "Cultivando Biodiversidad" (RAS) | Soberanía alimentaria | 


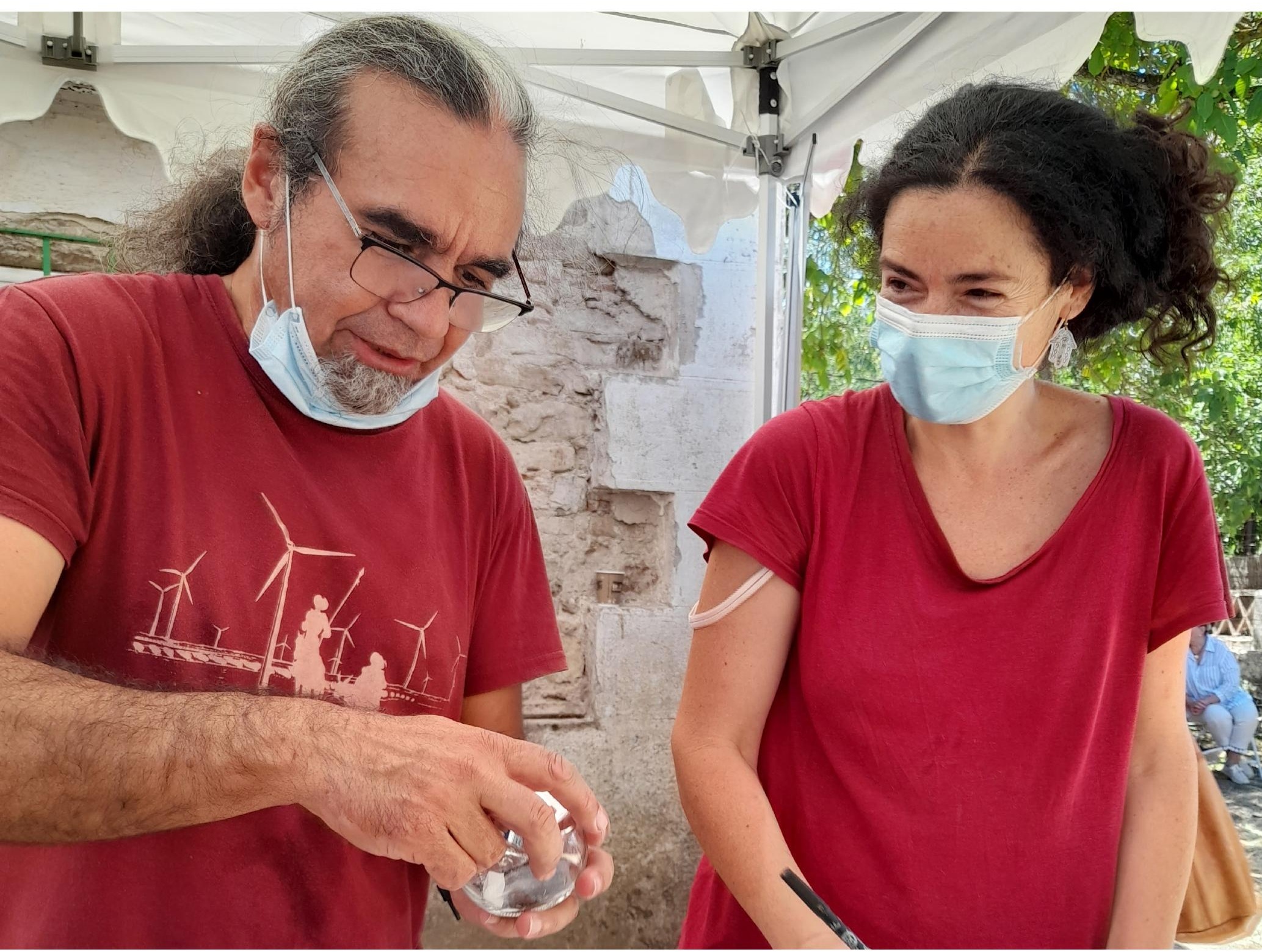

Intercambio de semillas de variedades locales en Cortes de la Frontera (Málaga) | foto Red Andaluza de Semillas "Cultivando Biodiversidad", quien ha facilitado todas las imágenes que ilustran este artículo 
La Red Andaluza de Semillas "Cultivando Biodiversidad" (RAS) es una organización de la sociedad civil que tiene entre sus fines contribuir a la recuperación de las variedades locales y el saber campesino tradicional, promover la gestión colectiva de la biodiversidad cultivada y fomentar los sistemas alimentarios campesinos y agroecológicos, como fuente de alimentos asequibles y saludables para todas las personas y como medio de vida digno para quienes los cultivan. La RAS se originó como diálogo de diferentes sensibilidades, prácticas y saberes que desde finales del siglo pasado han ido evolucionando y conformándose hasta nuestros días. En este diálogo han tenido un papel fundamental los agricultores locales, especialmente los vinculados al movimiento jornalero andaluz, que aportaron su visión crítica al sistema alimentario, y los hortelanos tradicionales, que nos han ido legando su formidable conocimiento en la gestión y manejo de la biodiversidad cultivada, y también el movimiento campesino internacional, que nos ha brindado un marco fundamental para la acción a través del concepto de soberanía alimentaria.

En este recorrido han tenido también un papel muy importante instituciones del mundo académico que, desde la reivindicación del campesinado y sus formas de conocimiento, dieron lugar a la agroecología como disciplina científica y como cuerpo de conocimientos prácticos que abordan integralmente la producción de alimentos desde un enfoque que promueve al mismo tiempo la sostenibilidad ambiental y la equidad social e intergeneracional.

Aunque desde sus inicios la RAS ha procurado nutrirse de ideas y saberes, su vocación es indudablemente la acción, dirigida a promover el uso de los recursos genéticos que permitan que la actividad de producción y elaboración de los alimentos siga siendo uno de los pilares del desarrollo sostenible local. Para ello nos coordinamos con otras organizaciones locales o globales que comparten nuestra misión hacia una agricultura en armonía con nuestro entorno y nuestra salud, en la que la biodiversidad cultivada y las comunidades agrarias que la desarrollan y gestionan tienen un papel fundamental.

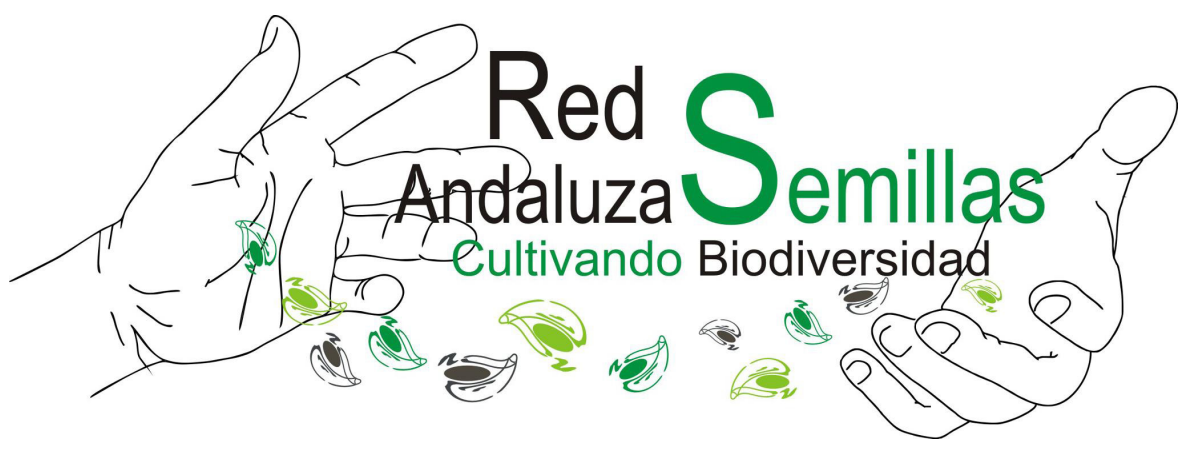




\section{LA BIODIVERSIDAD CULTIVADA COMO PATRIMONIO MATERIAL Y CULTURAL EN EVOLUCIÓN}

Las definiciones en el ámbito de la biodiversidad suelen ser complejas, ya que si bien siempre tienen como base el conocimiento científico, existen multitud de elementos que cobran peso en función del punto de vista y la finalidad de quienes las manejan.

En la práctica de la RAS, la biodiversidad cultivada es el elemento central. En este sentido la biodiversidad cultivada la conforman tanto los recursos materiales vivientes que utilizan los agricultores y agricultoras para perpetuar el sistema productivo de alimentos, como el cuerpo de conocimientos que permite su gestión y su adaptación a los cambios.

Tal como le ocurre a la propia agricultura, la biodiversidad cultivada desafía las clasificaciones clásicas de lo que se define como patrimonio, ya que participa a la vez de las características del patrimonio natural y cultural y también del patrimonio material e inmaterial. Estas cualidades y otras que trascienden este ámbito son las responsables de su riqueza y complejidad.

Desde el ámbito natural, el manejo de la biodiversidad está condicionado por los sistemas reproductivos de las plantas. Estos sistemas tienen en sí mismos una gran diversidad de manifestaciones desarrolladas a lo largo de la historia evolutiva de los vegetales y que hacen que existan formas vegetativas y sexuales de reproducción, como esquejes, bulbos o semillas por nombrar algunas de las más comunes.

En este mismo ámbito natural la biodiversidad cultivada posee a su vez un elemento informativo propio del material germinativo de los seres vivos que es la información codificada en su genoma. Los elementos reproductivos y la trasmisión de la información intergeneracional en las plantas está vinculado a sus formas de reproducción. Así en las formas de reproducción vegetativa esta información se conserva a medio-largo plazo de forma más o menos invariable, a excepción de mutaciones puntuales. Por el contrario, en el caso de la reproducción por semillas, la información genética trasmitida a los descendientes sufre una modificación respecto de los progenitores, que a su vez varía en función del grado de autogamia/alogamia de la especie y también, en cierto grado, de las estrategia floral (hermafroditismo, dioecia, etc.) y las formas de polinización (anemofilia, entomofilia, etc).

Con estas dimensiones naturales se entrecruzan los elementos culturales de manejo que están fuertemente condicionados a su vez por cuestiones materiales, como ocurre por ejemplo con las prácticas de conservación en función de las semillas, que pueden ser elementos de resistencia que permitan preservar durante un tiempo más o menos largo la viabilidad para generar un
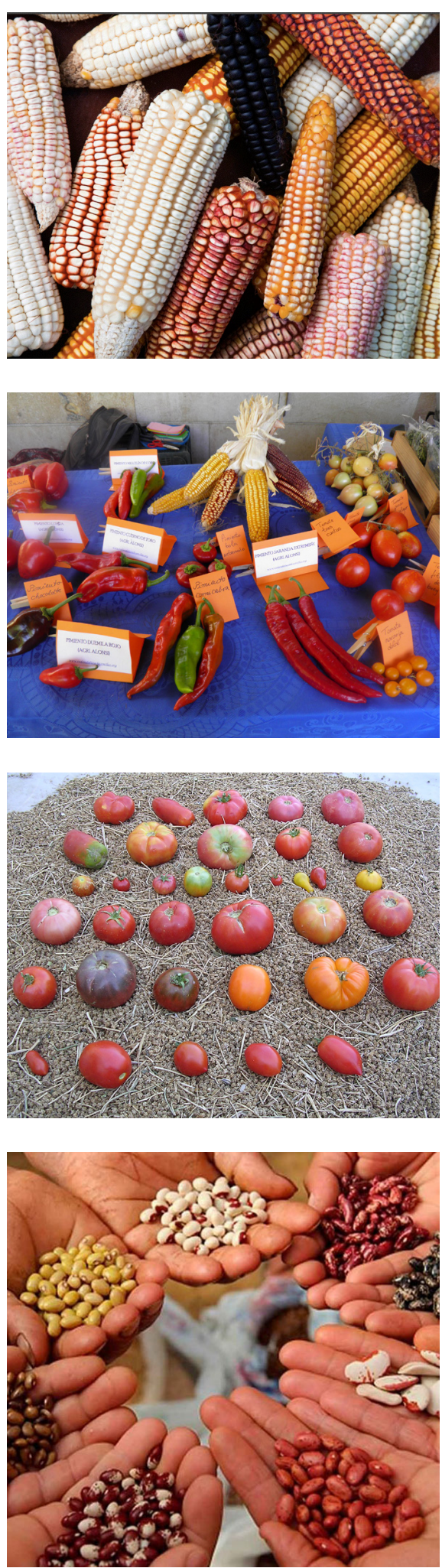
Encuentro de agricultores sabios durante trilla tradicional en Cuevas del Becerro (Málaga)

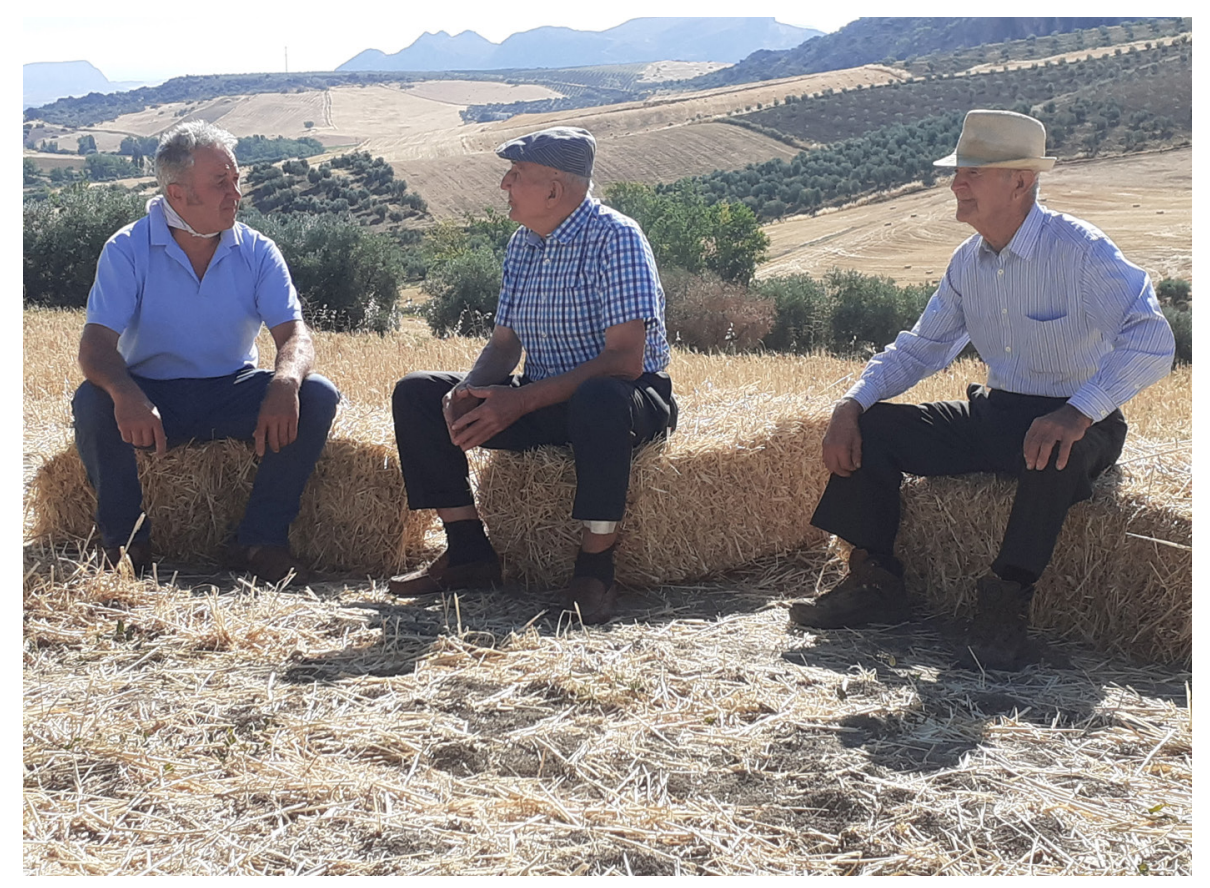

nuevo individuo o recalcitrantes que pierden su viabilidad germinativa en un periodo corto de tiempo.

A estos condicionantes materiales del elemento cultural se superpone a su vez una sabiduría campesina compleja sobre el manejo de las plantas que nos ha permitido a la humanidad sobrevivir y proliferar como especie durante cientos de años. Desde la RAS asumimos el reto de lograr el reconocimiento del conocimiento campesino andaluz como un conocimiento válido que ha asegurado la alimentación de nuestras comunidades a lo largo del tiempo y aún hoy sigue siendo esencial y también de racionalizar y reelaborar este conocimiento local de nuestros campesinos y campesinas para construir un nuevo edificio conceptual que sirviese de base para un modelo agroecológico de manejo de la biodiversidad cultivada (Soriano Niebla 2004).

Siendo conscientes de esta complejidad, desde la RAS hemos ido desarroIlando en nuestros 18 años de experiencia diversas iniciativas que vinculan el valor patrimonial de la biodiversidad cultivada con los fines de conservación y revitalización del patrimonio agrícola, la sostenibilidad de los modos de vida en el ámbito rural, la soberanía alimentaria y el equilibrio medioambiental y la protección de la biodiversidad.

Dentro de estas experiencias hemos tomado como ejemplo de buenas prácticas las aprendidas durante nuestra participación en el proyecto CERERE. Durante este proyecto hemos obtenido conocimientos sobre los múltiples 
aspectos que ligan los cereales tradicionales, su cultivo, su transformación y elaboración y su consumo desde una perspectiva actual.

El proyecto CERERE ha sido una iniciativa de 13 entidades europeas, aglutinando organizaciones de base del sector agrario, incluyendo a la RAS, e instituciones de investigación y de divulgación científica. Se desarrolló entre 2016 y 2019 y recibió financiación del programa de investigación e innovación Horizonte 2020 de la Unión Europea.

A través de CERERE hemos buscado una síntesis de prácticas innovadoras y los resultados de la investigación utilizados para construir sistemas alimentarios de cereales basados en la agrobiodiversidad, ecológicos y con bajos insumos. Para ello hemos trabajado con todos los actores implicados para encontrar las mejores prácticas existentes, los resultados más relevantes de la investigación y los casos de innovación y hemos impulsado su difusión.

Una de los motivos para incorporarnos a esta iniciativa fue compartir el enfoque de investigación participativo. Desde la práctica de la RAS el enfoque agroecológico se ha llevado a cabo a través de experiencias de investigación-acción participativa. Esto implica que el personal investigador profesional pasa a ser un agente mediador de la investigación, dando paso como sujetos a la comunidad que desarrolla y se beneficia de la investigación, que en nuestro caso la conforman los agricultores y agricultoras, las personas que producen el pan y la pasta y quienes se involucran en su consumo responsable.

La práctica de la investigación participativa implica también cambiar la realidad investigada de forma que la dialéctica entre la comunidad que investiga y el objeto de la investigación se plasma en una praxis de transformación colectiva para avanzar en la soberanía alimentaria local (Soriano et ál. 2013).

Para poder abarcar la complejidad que supone trabajar a la vez con la totalidad de actores relacionados con la valorización de los cereales tradicionales, se ha estructurado el trabajo en tres ámbitos: sistemas alimentarios alternativos y sostenibles, salud y nutrición, y agronomía y transformación alimentaria, lo que en primera instancia dio lugar a dos compilaciones sobre la literatura científica que apoyasen el desarrollo del proceso de investigación participativa (Sofi et ál. 2018; Sacchi et ál. 2018).

\section{BUENAS PRÁCTICAS EN EL CONOCIMIENTO Y MANEJO DE LA BIODIVERSIDAD CULTIVADA}

Para facilitar la comprensión de las interrelaciones entre la ecología y la producción se identificaron las especies de los principales cereales de invierno con presencia en los sistemas de cultivo tradicional en nuestro entorno 
1

DIVERSIFOOD es un proyecto del programa de investigación e innovación Horizonte 2020 de la Unión Europea Diversifood-High quality food systems.

Encuentro de agricultores sobre la recuperación de cereales antiguos en Alozaina Málaga (2012)

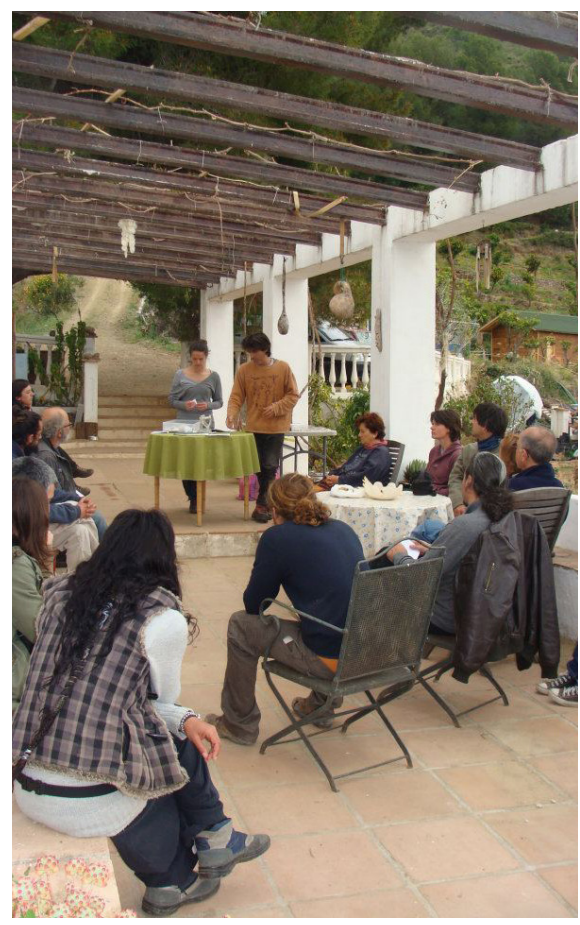

(escañas, escandas, espeltas, trigos duros y blandos, cebada, centeno y avena) y se describieron las características botánicas, así como la evolución de las diferentes especies y de la diversificación, dentro del género Triticum, entre los diferentes grupos en función de su poliploidía y su fruto desnudo o vestido.

Uno de los principales retos del trabajo con la biodiversidad cultivada es la descripción de las variedades para poder diferenciarlas, reconocerlas y, en trabajos más avanzados, llegar a su caracterización, incluyendo elementos relativos a su manejo, cualidades y formas de consumo.

Desde los inicios de la RAS se desarrollaron experiencias participativas de descripción y caracterización, sobre todo centradas en hortícolas y legumbres (Muñoz Pineda 2010; Toledo et ál. 2011a; Toledo et ál. 2011b; González et ál. 2014; Red Andaluza de Semillas 2012).

El trabajo de caracterización de variedades de trigo y asociaciones de cultivo locales se inició más tardíamente, a raíz de la participación en el proyecto europeo DIVERSIFOOD ${ }^{1}$. Las variedades locales de trigo que se describieron fueron Recio de Ronda, Chamorro de Los Portales, Capelli de Cañete, Trigo de Juan de Coín, Castellano, Chamorro de Albacete, Trigo del Corazón (Khorasan), Pichi de Antequera, Raspinegro de Jubrique, una accesión de trigo recio facilitada por el Centro de Recursos Fitogenéticos del INIA

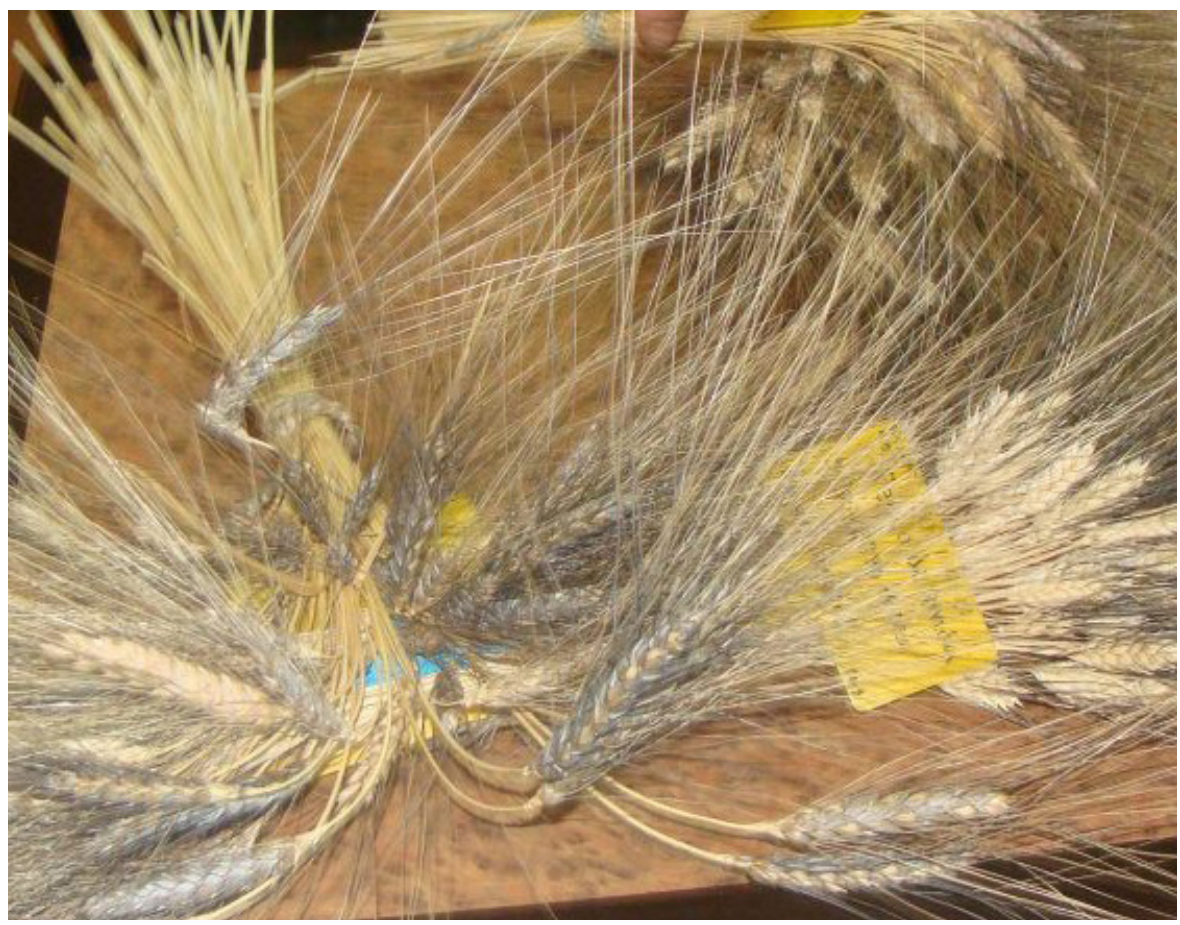


y la variedad comercial "Bronte". Los ensayos se llevaron a cabo en la fincas Ortuño, José Madalena y Antonio Campos en el municipio de Alozaina (Málaga) y en la finca Los Portales en Castilblanco de los Arroyos (Sevilla) (Carrascosa-García et ál. 2019).

Si bien las variedades tradicionales presentan una gran diversidad que permite adaptarse a las fluctuaciones de los sistemas de cultivo de secano, los sistemas agrícolas actuales y la demanda de productos locales no es la misma que la de hace unos años. Se hace pues necesaria continuar la mejora, integrando los conocimientos tradicionales practicados por los agricultores locales con las técnicas de selección vegetal desarrolladas a partir del redescubrimiento de las leyes de la genética.

La carencia de un sistema local de mejora, que integre ambas fuentes de conocimiento, había quedado patente en años anteriores a través de las experiencias desarrolladas en el marco del proyecto Farm Seed Opportunities ${ }^{2}$ (Valero et ál. 2010). Para paliar esta carencia, en la mejora participativa de trigos desarrollada en el proyecto CERERE se elaboró un protocolo para la selección de mezclas de poblaciones de cereales, dando la posibilidad a los agricultores/as de elegir las prácticas de selección en función de sus objetivos (van Frank 2018).

El trigo es una planta que en condiciones naturales se autopoliniza en un $95 \%$ y el polen restante se dispersa por medio del viento a las plantas cercanas, por lo que se dan pocos cruzamientos dentro de una población. Con el fin de aumentar la diversidad en caso de considerarlo necesario para la mejora se ha adaptado un protocolo para que los agricultores puedan fácilmente forzar de manera manual los cruzamientos (CERERE 2018a).

\section{BUENAS PRÁCTICAS EN EL PROCESAMIENTO Y ELABORACIÓN DE LOS PRODUCTOS BASADOS EN LA BIODIVERSIDAD LOCAL}

La puesta en valor de la biodiversidad cultivada está estrechamente vinculada a la recuperación de la demanda local. Mientras en algunos cultivos es posible la comercialización directa del producto, como pasa en el caso de hortalizas, frutales y legumbres, en el caso de los cereales su consumo se realiza en forma de pan y pasta, por lo que es necesario un proceso de transformación y elaboración.

Estos procesos tradicionales también han sufrido una importante erosión, habiendo sido sustituidos por otros industriales. En el caso de la molienda industrial el molido con piedras ha sido sustituido por el molido con cilindros metálicos que tiene mayor potencia, lo que afecta al contenido en salvado y germen de las harinas. 
Lo importante del proceso artesanal es realizar la molienda del grano entero, sin separar el germen ni todo el salvado, aportando mayor sabor y nutrientes a la harina. Generalmente la industria convencional prefiere harinas que no contengan germen porque se oxida con relativa rapidez y la enrancia, imposibilitando el almacenamiento durante largos periodos. Por el contrario, en panaderías artesanales se valora este tipo de harina, porque al ser frescas tienen mejores propiedades organolépticas y nutracéuticas que las que ya llevan tiempo procesadas.

En la panificación influyen diversos elementos, algunos de ellos derivados directamente de las propiedades de las variedades de trigo y otros relacionados con la masa madre utilizada en el proceso de fermentación y en las condiciones en las que se realiza el amasado, reposo y horneado. Para atender a estas cuestiones se han planteado soluciones basadas en la colaboración participativa de quienes hacen el pan con quienes cultivan el trigo y quienes lo consumen (Vindras-Fouillet y Serpolay-Besson 2020).

En Andalucía contamos con experiencias de panificación artesanal. Una de ellas es fruto de la colaboración entre la iniciativa Pan Para Todos y Más y la RAS. La molienda del grano la realizan en su propio molino eléctrico que trabaja con energía solar. La elaboración de pan sigue siendo de forma totalmente artesanal, amasados a mano, con masa madre y fermentación lenta y el horneado se hace en horno de leña. Disponen de su propia variedad tradicional de trigo Chamorro que les da singularidad a sus panes y experimentan con nuevas variedades tradicionales para mejorar su oferta (Chable et ál. 2019a, 26-32; Carrascosa et ál. 2019).

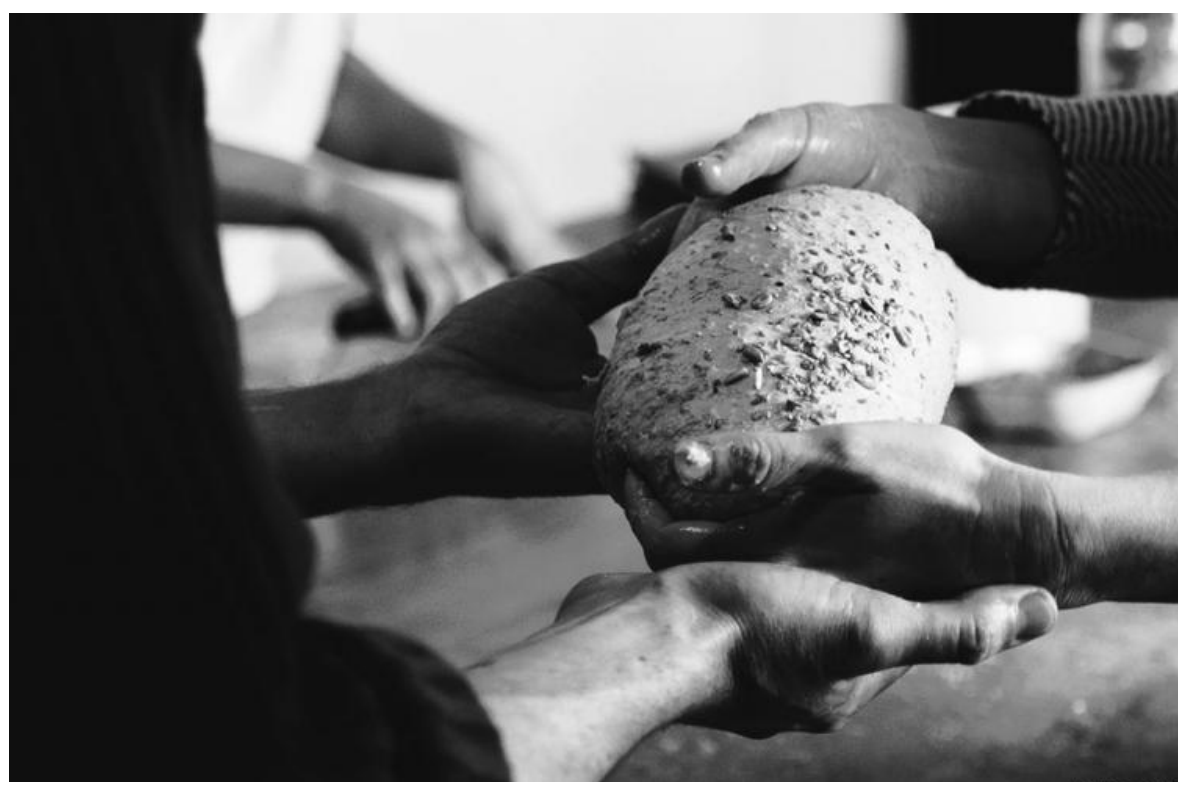




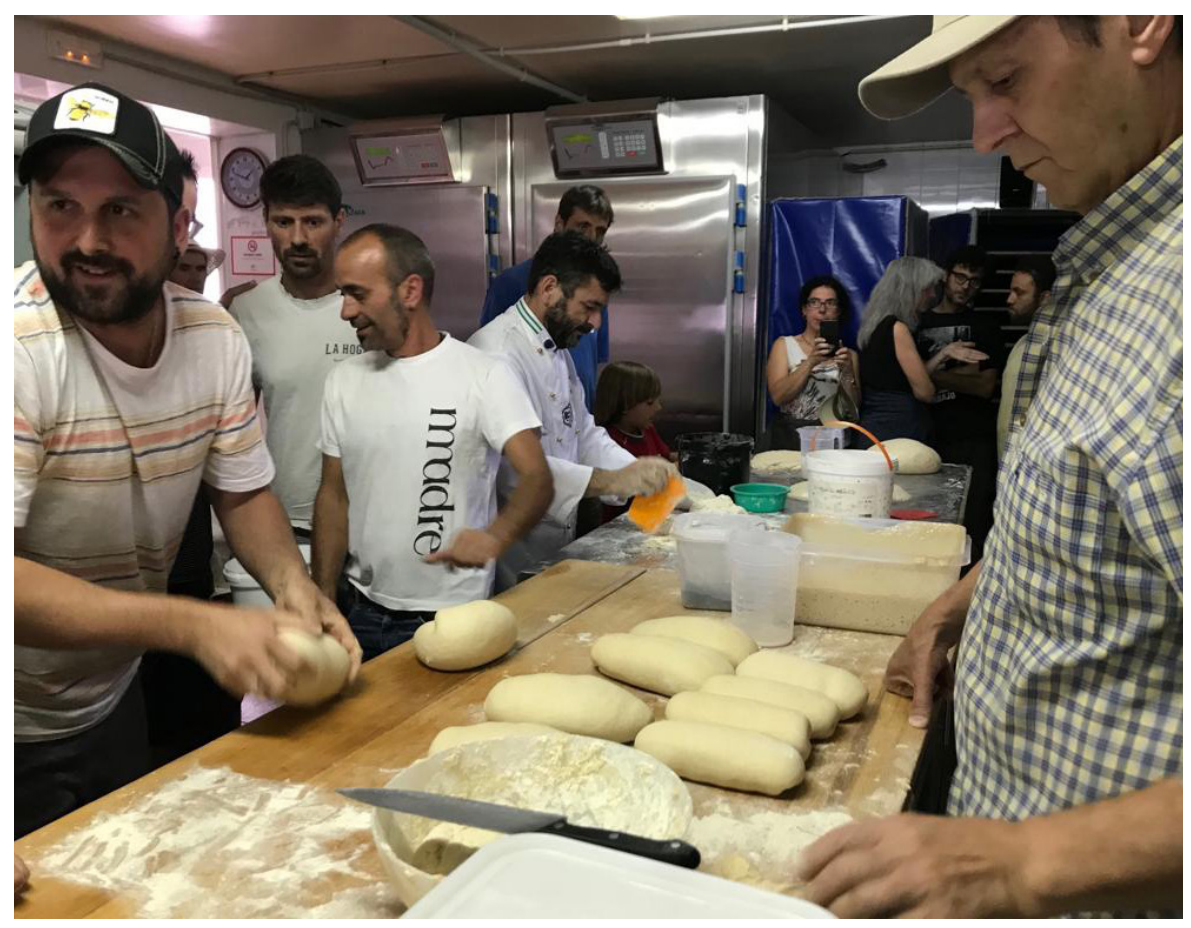

Otra experiencia se está desarrollando con La Artesa, panadería artesana situada en Sevilla, que ha incorporado dos variedades tradicionales (trigo duro Recio de Ronda y trigo blando Chamorro) para la elaboración de panes (Red Andaluza de Semillas 2018). En ambas iniciativas la comercialización de los panes se realiza a través de grupos de consumo, comercio local y ecomercados, esto permite generar un compromiso entre las partes implicadas, a nivel de cantidades y formatos suministrados y de precios adecuados a las diferentes necesidades.

También en la elaboración de pasta mediante procedimientos tradicionales se están desarrollando experiencias en Andalucía por parte de la empresa local Spiga Negra, un proyecto de elaboración artesanal de pasta. Spiga Negra cuenta con su propio molino ecológico y la infraestructura necesaria (prensa, moldes, armarios secadores). Para la elaboración artesanal de pasta de calidad siguen la tradición italiana, utilizando moldes de bronce que otorgan una rugosidad característica que permite un sabor más intenso al ser cocinadas. Posteriormente el secado se realiza de forma lenta, y a temperaturas siempre inferiores a los $40^{\circ} \mathrm{C}$. Un aspecto a destacar de esta iniciativa es el vínculo establecido con los productores y productoras de cereales con quienes planifican la producción y acuerdan los precios y cantidades de manera conjunta. Para ampliar las variedades de trigo que utilizan están colaborando con la Universidad Pablo de Olavide (UPO) en la evaluación de variedades (Chable et al. 2019b).
Taller de elaboración de pan con variedades locales de trigo en Cuevas del Becerro (Málaga) 


\section{BUENAS PRÁCTICAS PARA LA INTEGRACIÓN DE LA COMERCIALIZACIÓN Y EL CONSUMO EN EL CONOCIMIENTO Y VALORIZACIÓN DEL PATRIMONIO GENÉTICO AGRÍCOLA}

Uno de los actores colectivos con mayor trascendencia en el éxito de estas experiencias de conservación y revitalización del patrimonio cultural basadas en la biodiversidad cultivada lo constituyen los grupos de consumo. La RAS tiene una amplia trayectoria de trabajo con personas consumidoras de productos derivados de variedades locales a través de experiencias de evaluación participativa de variedades locales (Carrascosa et ál. 2012). La experiencia con trigos ha contribuido a mejorar la colaboración en el ámbito del consumo, organizando visitas a las fincas donde se han ensayado las variedades y recopilando información sobre la evaluación de la calidad de los panes tradicionales.

Para la evaluación de la calidad sensorial se ha adaptado un protocolo que permite aprovechar la experiencia de los panaderos expertos en la elaboración de masas (consistencia, fermentación ideal) hasta el horneado y poder formar a las personas consumidoras en el conocimiento de metodologías sensoriales. Para ello se ha partido del método del Napping, basado en una representación de la distancia sensorial, que se aplica y se modula constantemente a la luz de los resultados. Este test consiste en medir la distancia sensorial percibida entre los diversos panes por cada degustador. De los datos obtenidos, analizados en su conjunto, se desprenden las diferencias principales que perciben la mayoría de los degustadores (CERERE 2018b).

Otra intervención de los grupos de consumo que cada día adquiere una mayor relevancia son los sistemas participativos de garantía (SGP). Se trata de procedimientos acordados entre todos los intervinientes en la cadena de producción para certificar que el producto ha sido cultivado, procesado y comercializado siguiendo prácticas respetuosas con la producción local.

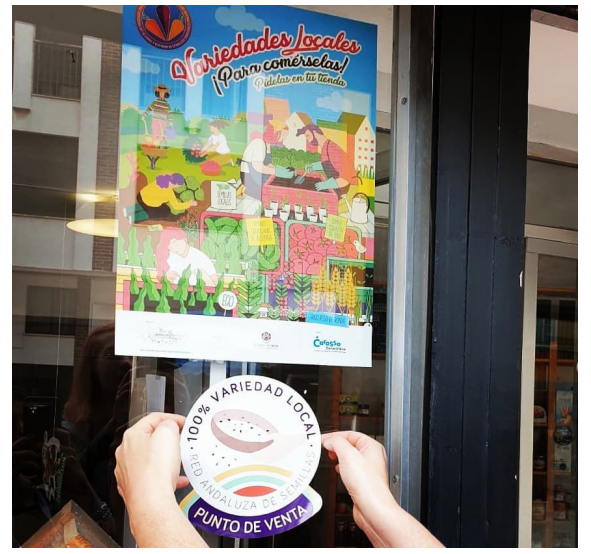
Esto permite crear distintivos identificables en los puntos de consumo que proporcionan confianza a quienes desean apoyar el desarrollo local y mejorar la calidad de su alimentación.

En uno de los estudios de caso de CERERE, se recoge una experiencia de sistema de garantía participativo, el de la Associazione Grani Antichi Montespertoli. Han desarrollado un sistema de control, basado en la participación activa de todas las partes interesadas (agricultores, molinero y panaderos), que es muy eficaz en el contexto local gracias a la confianza de los actores en el trabajo recíproco y a los mecanismos de construcción de la reputación fuera de la cadena. torno a una estrategia más amplia de comunicación para visibilizar y diferen- 


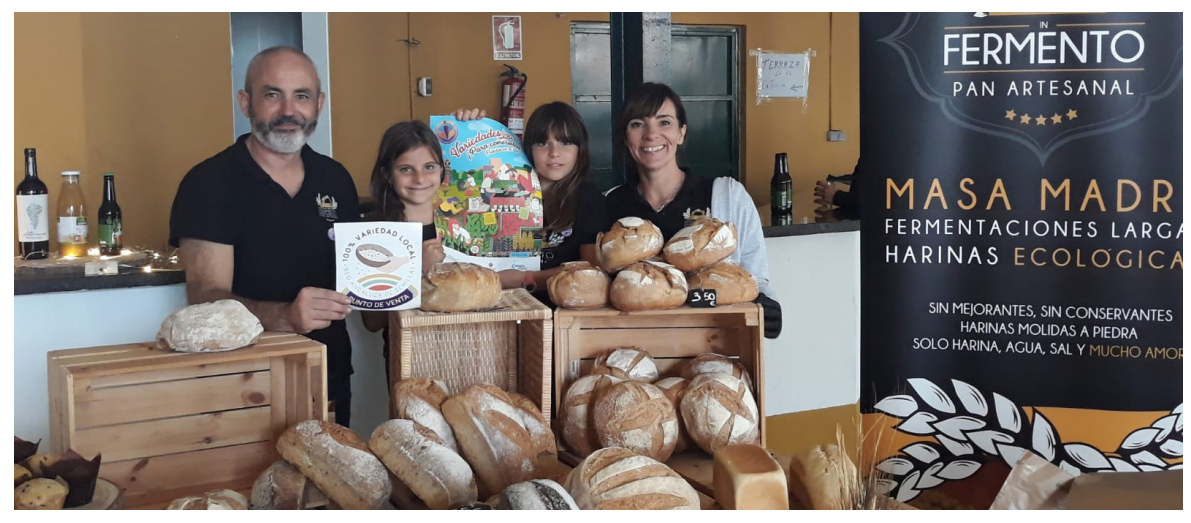

In Fermento, obrador de pan artesanal y ecológico

ciar los productos procedentes de variedades locales de cultivo disponibles en el mercado. Se ha creado el distintivo $100 \%$ variedad local como elemento clave para la valorización de la biodiversidad cultivada y se han establecido una serie de criterios protocolarios de uso: en qué tipo de variedades se va a utilizar, el tipo de origen de la semilla, los lugares a los que está destinado, etc. (Vara Sánchez et ál. 2020).

Los SGP representan un modelo de innovación social capaz de interpretar las necesidades de los territorios locales que a menudo las normativas nacionales y/o europeas no consiguen reflejar. De hecho, el desarrollo de los SGP facilita varios procesos sociales que, por una parte, favorecen la inclusión social y la cohesión al reforzar las relaciones de confianza entre los pertenecientes a redes agrícolas y, por otra, crean un círculo virtuoso en el que las reglas de la red y el conocimiento recíproco invitan a implantar un sistema de garantía eficiente (CERERE 2019).

\section{A MODO DE CONCLUSIÓN}

El mantenimiento sostenible de los agroecosistemas requiere generar un nuevo conocimiento que integre los avances científicos y técnicos en el ámbito de la agronomía con el conocimiento tradicional desarrollado localmente por generaciones de agricultores y agricultoras.

Este camino pasa necesariamente por intensificar la investigación participativa en el ámbito de la agroecología, fortaleciendo la cooperación entre los organismos de investigación y las organizaciones de base comprometidas con el desarrollo local y la soberanía alimentaria.

Como se ha intentado ilustrar en este capítulo, el fruto de esta colaboración, se traduce en buenas prácticas que permiten poner en valor nuestro extenso patrimonio genético agrícola. 


\section{BIBLIOGRAFÍA}

- Carrascosa, M.; J.M. González, L. Toledo, J.J. Soriano, P. López, T. García-Muñoz, P. González y I. Sanz (2012) Recuperación de variedades tradicionales, una estrategia combinada de conservación de la biodiversidad agrícola, agroecología y desarrollo sostenible del medio rural en Andalucía. En: Labrador, J. y Gonzálvez, V. (coord.) Actas del $X$ Congreso de SEAE "20 años impulsando la producción ecológica". Albacete, 26-29 septiembre 2012. Valencia: SEAE-Sociedad Española de Agricultura Ecológica. Disponible en: https://www.agroecologia.net/ recursos/publicaciones/actas/cd-actas-xcongresoseae/ index.html [Consulta:05/07/2021]

- Carrascosa-García, M., Soriano Niebla, J.J., Navarro Chaves, A., Toledo, L., Roldán, E., Fulgado, J.A., González, P., González, M., Martínez, I. y Ceccarelli, S. (2019) Evaluación participativa de variedades locales de trigo y tomate en Andalucía. Red Andaluza de Semillas. Disponible en: https://www.redandaluzadesemillas.org/ sites/default/files/recursos/2019/190821\%20Informe\%20 DIVERSIFOOD\%20 evaluacion $\% 20$ trigos $\% 20 y \% 20$ tomates.pdf [Consulta:05/07/2021]

- Chable, V., Ducottet, Ch., Kutelmach, M. Flipon, E., De Santis, G. Rodríguez, I. (ed.) (2019a) Biodiversidad agrícola: historias de éxito en Europa. CERERE Project. Disponible en: http://cerere2020.eu/wp-content/uploads/2021/01/CERE RE_Book_Case_studies_SPANISH.pdf [Consulta: 06/07 /2021]

- Chable, V., Ducottet, Ch., Kutelmach, M. Flipon, E., De Santis, G. Rodríguez, I. (2019b) Spiga Negra, una iniciativa local de producción de pasta artesanal y ecológica. En: Biodiversidad agrícola: historias de éxito en Europa. CERERE Project. Disponible en: http://cerere2020.eu/wpcontent/uploads/2021/01/CERERE Book Case studies SPANISH.pdf [Consulta:06/07/2021]

- CERERE [CEreal REnaissance in Rural Europe] (2018a) Cruzamientos manuales para impulsar la diversidad genética de las poblaciones de cereales. Ficha informativa sobre innovación CERERE \#9, marzo 2018. Disponible en: http://cerere2020.eu/wp-content/uploads/2020/03/9 ES.pdf [Consulta:06/07/2021]

- CERERE (2018b) Estudio sobre la calidad de los panes campesinos. Ficha informativa sobre innovación CERERE \#6. septiembre 2018. Disponible en: http:// cerere2020.eu/wp-content/uploads/2020/03/6_ES.pdf [Consulta:06/07/2021]

- CERERE (2019) Los sistemas de garantía participativa (sgp) para valorar y asegurar la autenticidad de las cadenas de cereales antiguos. Ficha informativa sobre innovación CERERE \#19, marzo 2019. Disponible en: http://cerere2020.eu/wp-content/uploads/2020/03/19 ES.pdf [Consulta:06/07/2021]
- González, J., Carrascosa, M., Soriano, J., GarcíaMuñoz, T., Toledo, L., López, P., Hidalgo, J., y Navarro, A. (2014) Investigación-Acción Participativa como herramienta de empoderamiento: El caso de la descripción participativa de variedades tradicionales en las redes de intercambio de semillas. En: Herrera Molina, F., Tarifa García, F. y Hernández Bermejo, E. (ed.): ICEB 2014 : VI Congreso Internacional de Etnobotánica, 17-21 de Noviembre, Córdoba. Córdoba : IMGEMA, Jardín Botánico de Córdoba

- Muñoz Pineda C. (2010) Caracterización de variedades locales hortícolas andaluzas. TFMáster de Agricultura Ecológica inédito, Universidad de Barcelona. Disponible en: https://www.redandaluzadesemillas.org/sites/default/files/ recursos/2020/proyecto\%20UB.pdf [Consulta:05/07/2021]

- Red Andaluza de Semillas (2012) Informe Descripción de variedades tradicionales andaluzas en fincas agroecológicas de Sevilla, Córdoba, Cádiz y Málaga. Temporada Primavera-Verano 2012. Disponible en: https://www.redandaluzadesemillas.org/sites/default/ files/recursos/2020/121231_informe\%20descripcion\%20 VL\%20en\%20Sevilla\%20Cordoba\%20Cadiz\%20y\%20 Malaga.pdf [Consulta:05/07/2021]

- Red Andaluza de Semillas (2018) Elaboración de pan artesano y ecológico con variedades tradicionales de trigo duro y trigo blando. Resumen de prácticas CERERE \#2, mayo 2018. Disponible en: http://cerere2020.eu/wpcontent/uploads/2019/01/211205-2PA-Baking-traditionalvarieties-RAS-ESP.pdf [Consulta:06/07/2021]

- Sacchi, G., Cei, L., Stefani, G., Lombardi, G.V., Rocchi, B., Belletti, G., Padel, S., Sellars, A., Gagliardi, E., Nocella, G., Cardey, S., Mikkola, M., Ala-Karvia, U., MackenWalsh, À., McIntyre, B., Hyland, J., Henchion, M., Bocci, R., Bussi, B., De Santis, G., Rodriguez y Hurtado, I., De Kochko, P., Riviere, P., Carrascosa-García, M., Martínez, I., Pearce, B., Lampkin, N., Vindras, C., Rey, F., Chable, V., Cormery, A. y Vasvari, G. (2018) A Multi-Actor Literature Review on Alternative and Sustainable Food Systems for the Promotion of Cereal Biodiversity. Agriculture, 2018, vol. 8, n. ${ }^{\circ} 11,173$. Disponible en: https://www.mdpi.com/20770472/8/11/173 [Consulta: 08/09/2021]

- Soriano Niebla, J.J. (coord.) (2004) Hortelanos de la Sierra de Cádiz. Las variedades locales y el conocimiento campesino sobre el manejo de los recursos genéticos. Villamartín, Cádiz: Mancomunidad de municipios de la Sierra de Cádiz

- Soriano, J.J., Carrascosa, M., González, J.M., García, T. y Sanz, I. (2013) Mejora agroecológica participativa (MAP) y biodiversidad agrícola. Aplicación de la investigación-acción participativa al manejo de las variedades tradicionales en Andalucía. Agroecología, vol. 7, n. ${ }^{\circ}$ 2, pp. 21-30. Disponible en línea: https://revistas.um.es/agroecologia/article/view/ 182821 [Consulta: 05/07/2021] 
- Sofi, F., Dinu, M., Pagliai, G., Cei, L., Sacchi, G., Benedettelli, S., Stefani, G., Gagliardi, E., Tosi, P., Bocci, R., Bussi, B., De Santis, G., Rodriguez y Hurtado, I., De Kochko, P., Riviere, P., Carrascosa-García, M. y Martínez, I. (2018) Health and Nutrition Studies Related to Cereal Biodiversity: A Participatory Multi-Actor Literature Review Approach. Nutrients 2018, 10(9), 1207. Disponible en: https://doi.org/10.3390/nu10091207 [Consulta: 05/07/2021]

- Toledo, L., González, J.M., Carrascosa-García, M., y López, P. (2011a) Informe Huerta experimental. Red de Resiembra e Intercambio de variedades locales de cultivo. Temporada Otoño-Invierno 2010-2011. Sevilla: Red Andaluza de Semillas. Disponible en: https:// www.redandaluzadesemillas.org/sites/default/files/ recursos/2020/Informe_RAS_Huerta_Experimental_OI_2010-2011_01feb2011.pdf [Consulta: 05/07/2021]

- Toledo, L., González, J.M., Muñoz, C., Soriano, J.J., y García-Muñoz, T. (2011b) Informe de la Huerta experimental de la Red de Resiembra e Intercambio de variedades locales de cultivo. Temporada Primavera-Verano 2011. Sevilla: Red Andaluza de Semillas

- van Frank, G. (2018) Gestion participative de la diversité cultivée et création de mélanges diversifiés de blé tendre à la ferme. Génétique des populations. Tesis doctoral inédita, I'Universitè Paris-Saclay. Disponible en: https://tel.archivesouvertes.fr/tel-02301993/document [Consulta: 06/07/2021]

- Valero, T., González, J.M., Soriano, J.J. y López, P. (2010) Oportunidades para la conservación, mejora y producción de las semillas campesinas. En: Gonzálvez, V. (coord.) Actas del IX Congreso de SEAE: Calidad y seguridad alimentaria. Lleida, 6-9 de octubre 2010. Valencia: SEAESociedad Española de Agricultura Ecológica, pp. 226-238. Disponible en: http://orgprints.org/29758/1/actas-Ileida-vd. pdf [Consulta: 26/07/2021]

- Vara Sámchez, I., Soriano Niebla, J.J., Gallar Hernández, D. y Iglesias Losada, B. (2020) Variedades locales en Andalucía. Debates y recomendaciones para fomentar su producción, comercialización y consumo en sistemas alimentarios sostenibles. Sevilla: Red Andaluza de Semillas. Disponible en: https://www.redandaluzadesemillas.org/sites /default/files/recursos/2020/Variedades\%20locales\%20 en\%20Andalucia\%20Debates\%20y\%20recomendaciones. pdf [Consulta: 26/07/2021]

- Vindras-Fouillet, C. y Serpolay-Besson, E. (2020) Recopilación de soluciones para la panificación. Booklet \#3, CERERE Project. Disponible en: http://cerere2020.eu/ wp-content/uploads/2020/06/Booklet3_ESP.pdf [Consulta: 06/07/2021] 